\title{
Study on Bel Canto in Singing Skill Training
}

\author{
Hui Zhang \\ Ankang University, Ankang Shaanxi, 725000, China
}

Keywords: Singing skill training, Bel canto, Bel canto principle, Key training point

\begin{abstract}
Bel canto, also called as soft singing technique, requires singers to use half power for singing, not to use strong breath for impact especially in high-pitched singing process, and use natural and soft tune for singing to make the voice smooth. In singing skill training, bel canto has certain personalized features compared to other singing techniques and its training method is obviously different. This paper analyzes bel canto principles in singing skill training based on brief introductions to singing skill training, points out key training points of bel canto in singing skill training and expects to promote further development of bel canto.
\end{abstract}

\section{Introduction}

Due to differences of physical quality, each singer has different voice conditions. Meanwhile, due to the influence of conditions and social living environment, different people have corresponding differences in the singing mode and representation style of the same song. Singing technique system of music art naturally presents different development situations. However, for whichever training mode, it is required to conduct appropriate singing skill training and follow corresponding singing technique principles. This paper conducts appropriate research on bel canto in singing skill training and expects to provide certain guidance for singers, improve their singing ability and make corresponding contributions to the development of Chinese vocal music art.

\section{Singing skill training}

Singing skill training generally contains breathing, sounding, pronunciation and listening training which are closely connected and promote each other in actual training process and form an organic integrity of dynamic singing skill training. During singing skill training, singers should give consideration to training contents consciously, establish a reasonable training plan by combining different training conditions and practice and avoid one-sided training to the greatest extent and adverse influence on the training of singing skills. Meanwhile, singers should also pay attention that singing skill training cannot be simply considered as exercise of body movement. More importantly, it trains command capacity of brain for body and coordination ability of each part of body. Breathing, sounding, pronunciation and listening training contents are influenced by brain dominance ability. Therefore, singers must keep a cool head in actual training process, be good at discovering problems existing in singing training process, positively explore corresponding measures for solving problems and gradually improve their singing skills. In addition, singers have some common problems in singing training, such as excessive tension for breathing and sounding, which seriously influence training effect. Singers should pay attention to train their singing awareness, overcome problems existing in singing process, coordinate the movement of each organ of body, improve their singing skills and bring high-quality music art enjoyment to audiences.

\section{Implementation of bel canto principle in singing skill training}

In singing skill training, particular specification of bel canto generally contains two meanings, i.e. vertical singing technique in terms of physiological mechanism and horizontal singing technique at the level of consciousness and feeling. Vertical singing technique is formed based on singing technique principle. Vertical singing technique is naturally produced when singers' sound source, breath and sound track are correspondingly supported in actual singing process. Horizontal singing 
technique is generally used to control and coordinate with vertical singing technique. This basic theory fully shows that bel canto in singing skill training mainly involves two modes - with overall and fringe vibration vocal cords as subject and with local vibration vocal cords as subject. In practical singing process, the correct state of feeling of bel canto is sound from up to down and breathing from bottom to top.In singing skill training, bel canto uses such opposite awareness to provide continual power for the stability of vertical singing technique and horizontal movement of sound. Therefore, principles of bel canto can be implemented in the following aspects in the process of singing skill training.

\section{Constitute head voice with "points" gathered in singing}

Closure and concentration in bel canto represent head voice. Simply speaking, head voice is a special voice product produced by ecological fringe sounding vibration and the initial point of all relevant music performance skills as well as the seed of bel canto. In singing skill training, closure and concentration skills show points of voice through appropriate gathering of voice and such points have certain changes with the melody in the specific singing process, gradually turn into a line and finally form vocal line. Each sound area originally split up is integrated and form a unified integrity. This singing skill requires the training of bel canto with the following method: singers find the gathering point of voice by auditioning with $\mathrm{Hn}$ and hum, guarantee to place voice behind incisor when starting voice and start the voice softly to discover the gathering point tentatively. For singers, the scale of sound gathering point does not have a unique standard, which can be loud or quiet. It will be right as long as the vocal cord of singers is kept in stop state.

\section{Obtain singers by yawn}

In singing skill training, openness and resonance mainly refer to the openness of resonance chamber. Singers can open resonance chamber smoothly only by guaranteeing the stability of their throat. Only by placing throat down can they really open the throat and produce high-quality resonance effect. The physiological phenomenon of yawn can well help singers solve the practical problem. When a singer is yawning, his nasopharynx and laryngopharynx are strained and throat will be inevitably placed down, thus producing ideal resonance [1].For the specific training method, singers find the gathering point of voice through Hxx and then impel throat to yawn in the position of the gathering point, thus making voice produce resonance and improve the singing effect of bel canto. In the training process, attention should be paid that mandibula of singers must be relaxed, thus guaranteeing actual effect of resonance.

\section{Obtain breathing support through voice starting with whisper}

Obtaining the support of singing breathing with corresponding expiration method is an important link in the adjustment of singing function. To obtain a good effect in bel canto, it is most important to draw voice and breath close and achieve the effect of integrating it with sound source and track. Therefore, the exercise of bel canto must be started in two stages in singing skill training: first, the exercise of beginners with whisper can impel the support of sound source and breath and sound track to form an effective connected operating mechanism, allow them to naturally feel the operating law of the mechanism in the learning process and then learn to control breath, adjust physical function appropriately and achieve the best singing effect. Second, singers who have mastered the adjustment of singing function can create the corresponding feeling with voice starting with whisper and then achieve the effect of strengthening breathing support. The application of this method can effectively strengthen the support of breathing, achieve an effect of voice from far to near and improve the singing level of singers.

\section{Bel canto in singing skill training}

In many countries and nationalities in the world, singing method has certain differences due to the influence of conditions of singers. The selection of different singing methods depends on the method used for sounding of organ. Over the centuries, people have constantly conducted practical exploration, enrichment and innovation in the pursuit for singing sound and gradually established a bel canto system meeting the law of scientific sounding. They can develop their resonant cavities to 
the greatest extent by organically coordinating each relevant vocal organ of human body, strive to achieve the best singing effect with minimum energy, impel sound vigor and softness to highly coordinate with each other and finally produce a strong artistic charm of voice of singers, arouse the resonance of audiences and achieve a high aesthetic value [2].In this relatively scientific bel canto singing system, the support of breathing, development of resonant cavity and openness of throat are parts of core training contents. Their relevant factors depend on and restrict each other. As long as the balanced development of each factor is guaranteed, the overall balance can be maintained and voice quality can be influenced.

For bel canto in singing training, singing art is same as breathing art to a certain extent. Breathing is the source of singing as well as the foundation for obtaining singing power. Correct breathing method has vital influence on coordinated movement of singing organs and the improvement of voice quality of singers. United respiration of chest and abdomen in bel canto in singing training can fully exert the power of muscle groups such as diaphragmatic muscle, abdominal muscle and costal muscle of singers, strengthen their breathing, show certain elasticity and guarantee respiratory capacity correspondingly. The cooperation of muscle groups can form a countervailing power invisibly, produce a point of breathing, make singing organs more coordinated, improve singing elasticity of singers and bring audiences a special aesthetic feeling.

Meanwhile, as people using bel canto gradually realize the influence of throat on singing quality in the practice of singing skill training, bel canto training strengthens the control over throat. For singers of bel canto, throat is one of the important component elements of sound source, which is located at the junction of chest resonance chamber and head resonance chamber. It makes cavum pharyngis from throat to soft palate become an important resonance cavity of bel canto to a certain extent. For singers of bel canto, opening throat in training means lowering throat and lifting up soft palate to further expand the area of resonance chamber of cavum pharyngis and guarantee actual resonance effect of cavum pharyngis ${ }^{[3]}$.In addition, when singers open the throat, $20 \%$ energies emitted by vocal cord will realize resonance of cavum pharyngis and other parts will smoothly enter other resonance chambers, thus greatly expanding the effect of overtone and impelling singers to obtain the best vibration effect in the singing process.

Moreover, resonance in bel canto has significant influence on singing tone, volume and tone quality. Therefore, singing skill training should attach more importance to training in these aspects. In bel canto, the development of resonance chamber refers to maximum development of nonadjustable and adjustable resonance chambers on the body of singers, further expansion of resonance chamber, increase of volume and improvement of overtone effect, thus making the sound of singing softer and graceful and improving the infection of singing art ${ }^{[4]}$. Meanwhile, besides the use of yawn training method mentioned for opening mouth and pharyngeal resonance chamber in actual singing process, it is also necessary to use certain method to open pectoral and nose resonance chambers, keep an excitatory state between the eyebrows and then make nonadjustable resonance chamber of singers keep a corresponding positive state. Only in this way can adjustable and nonadjustable resonance chambers of singers realize effective connection and integration and form a mixed resonance system. Mixed resonance is the foundation for singers of bel canto to improve their singing quality, which can organically integrate the resonance produced by singers' personalized tone in actual application process and form a universal resonance effect. Therefore, appropriate development of resonance chamber must regard human body as a relatively complete resonance chamber and attach importance to the development of each part in entirety so as to explore the potential of resonance and exert functions of resonance to the greatest extent.

To guarantee the effect of bel canto in singing skill training, an important standard should be reached, i.e. from low pitch to high pitch. Tone, tone quality and volume should be harmonious and unified. Each sound area can realize natural transition without obvious trace of transition. Generally, certain falsetto is added in middle pitch part to achieve the effect of mixed voice in male and female voices of bel canto. The proportion of falsetto increases with the constant increase of volume after entry into high pitch part, thus making the sound of singers wrapped by generous breathing, being full and manifesting certain elasticity and improving the attraction of voice to audiences. Such effect of 
tone will be maintained in each different sound area, especially after singers gradually enter high pitch part from voice change area in the performance process ${ }^{[5]}$. When head cavity is used as the main resonance chamber in high pitch part, to guarantee the singing effect, singers need to coordinate mouth, cavum pharyngis and chest. When cavum pharyngis resonance is used as the subject in middle pitch part, it is necessary to realize the resonance of head cavity and chest. When chest is the main resonance body in low pitch part, the resonance of cavum pharyngis and head cavity seems especially important. It is thus clear that such integrated training and use of real tone and falsetto and the formation of mixed resonance can obviously make the voice of singers more smooth, realize traceless transition, form a high-quality sound area and obtain excellent singing effect.

Therefore, the application of bel canto in singing skill training should guarantee normal movement of each vocal organ and coordination of relevant organs. To achieve the best training effect, singers should exercise continuously for a long term so as to make wonderful and nice sound in actual singing process, improve the infection of bel canto awareness and impel bel canto to achieve better development.

\section{Conclusion}

In conclusion, this paper focuses on analyzing bel canto in singing skill training. In singing skill training, to further improve the actual application quality of bel canto, it is required to strengthen the control over physical function, coordinate the movement relation of each part of body, exert functions of body movement to the greatest extent, improve the application effect of bel canto and create conditions for the improvement of singers' singing level.

\section{References}

[1] Chen Yali. Brief Discussions on Manifestation of Bel Canto Principle in Singing Skill Training. Notonly Music, 2014(12):171-171.

[2] Chen Yanhe. Brief Discussions on Characteristics and Method of Sound Production of Bel Canto. Yellow River of the Song, 2015(4):73-74.

[3] Yang Fengling. View on Development of Bel Canto from Musical Theater. Art Review, 2012(5):107-109.

[4] Zhang Shu. Brief Discussions on Singing Skills and Method of Sound Production of Bel Canto. Family Education World, 2012(8):130-131.

[5] Liu Xiaolin. Matters Needing Attention in Singing Voice Training - Use System Concept of Bel Canto to Improve Singing Art. Music Space Time, 2014(24):173-173. 